\title{
Novel Method for Multiturn Extraction: Trapping Charged Particles in Islands of Phase Space
}

\author{
R. Cappi and M. Giovannozzi \\ CERN, PS Division, CH 1211 Geneva, Switzerland \\ (Received 18 May 2001; published 19 February 2002)
}

\begin{abstract}
A novel method for multiturn extraction from a circular particle accelerator is presented, based on trapping particles into islands of phase space generated by nonlinear resonances. By appropriate use of sextupoles and octupoles, stable islands can be created at small amplitude in phase space. By varying the linear tune, particles can be trapped inside these islands and then transported towards higher amplitudes for extraction. Results of numerical simulations are discussed.
\end{abstract}

DOI: $10.1103 /$ PhysRevLett.88.104801

PACS numbers: 29.27.Ac, 05.45.-a, 29.27.Bd

Charged particles can be extracted from a circular machine by two different methods: (i) fast extraction, (ii) slow extraction. In the first case, the whole beam is ejected in one turn by means of fast dipole (kicker) and septum magnets. This technique can be used for transferring the beam either to a subsequent machine or towards a target for physics experiments. The latter method is based on the effect of a third-order resonance [1,2]: unstable motion generated by the separatrix joining the three unstable fixed points increases the particles' amplitude. This technique allows beam extraction over many machine turns (typically many thousands), and it is only used when delivering the beam to a target for physics experiments.

In some special cases, an intermediate extraction mode, called multiturn extraction, is needed. This is the case for the transfer between the CERN Proton Synchrotron (PS) and the Super Proton Synchrotron (SPS). The two machines have different circumferences, satisfying the relation $C_{\mathrm{SPS}}=11 C_{\mathrm{PS}}$. Hence, to fill the SPS, one would require ten fast-extracted pulses from the PS (the empty gap in the SPS is needed for the rise time of the injection kicker). If the filling time has to be minimized, then the solution consists of extracting the beam over a few turns. In practice, a five-turn extraction was proposed, allowing the SPS to be filled with only two PS pulses. Such an approach is called Continuous Transfer (CT) [3]. In Fig. 1 the layout of the extraction elements is shown together with the horizontal normalized phase space. Just before extraction, the horizontal tune is set to the value 6.25 and the closed orbit is modified so that the blade of an electrostatic septum intercepts the beam. Four slices are shaved off the main core and extracted as a continuous ribbon over four turns. The central part is extracted last, during the fifth turn, by changing the beam trajectory so as to jump over the septum blade. In addition to the septum used to slice the beam, a kicker and a magnetic septum are used for the extraction. Another interesting property of such an approach is that the horizontal emittance of the extracted beam is decreased with respect to that of the circulating one. However, a number of drawbacks are present, namely, (i) beam losses, especially at the electrostatic septum, are unavoidable. They amount to about $10 \%-15 \%$ of the total beam intensity. (ii) The extracted slices do not match the natural foliation of phase space into circles, thus generating a betatronic mismatch. This, in turn, induces emittance blowup in the receiving machine. (iii) The extracted slices have different transverse emittance.

These points mean that the CT extraction is not very suitable for the planned CERN Neutrino to Gran Sasso beam [4]. This is a high-intensity proton beam: in its nominal version, the PS should deliver two pulses of more than $3 \times 10^{13}$ protons each to the SPS. Efforts are being made to study a possible intensity upgrade by a factor of about 2. In the new scenario, the beam losses related to the present scheme of the five-turn CT would not be acceptable, and the properties of the extracted beam would not allow an efficient injection into the SPS.

In the novel approach, nonlinear elements such as sextupoles and octupoles are used to generate stable islands in transverse phase space. Then, by varying the horizontal tune, particles can be selectively trapped in the islands by adiabatic capture: some will remain in the phase space area around the origin, while others will migrate to the stable islands. As a result, the beam is split into a number of parts in transverse phase space, determined by the order of the resonance used, without any mechanical action. Finally, it

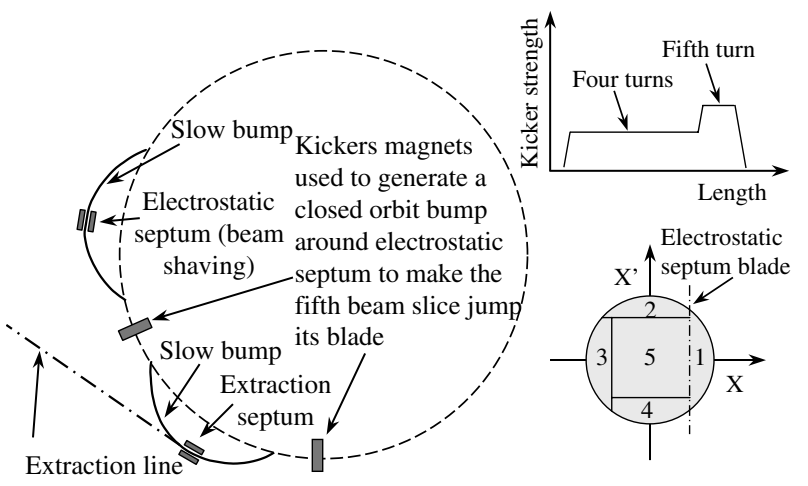

FIG. 1. Principle of the CT extraction from the PS machine. The schematics of the extraction elements is shown on the left, while in the upper right part the kicker strength as a function of time is shown. In the lower right part the normalized phase space is depicted. 
is possible to move the particles trapped inside the islands towards higher amplitudes. This increases the separation between the different slices so that enough room is available for the beam to jump over a septum blade with almost no particles lost.

The principle of this novel approach has been tested using a simple model representing the horizontal betatron motion in a circular machine under the influence of sextupole and octupole magnets. The motion in the vertical plane can be safely neglected for our purposes. The nonlinear magnets are assumed to be at the same location in the ring and are represented in the single-kick approximation (see Ref. [5] for more details). The one-turn transfer map can be expressed as $\mathbf{x}_{\mathbf{n}+\mathbf{1}}=\mathbf{M}_{\mathbf{n}}\left(\mathbf{x}_{\mathbf{n}}\right)$, or

$$
\begin{gathered}
\left(\begin{array}{c}
x_{n+1} \\
x_{n+1}^{\prime}
\end{array}\right)=R\left(2 \pi \nu_{n}\right)\left(\begin{array}{c}
x_{n} \\
x_{n}^{\prime}+x_{n}^{2}+\kappa x_{n}^{3}
\end{array}\right), \\
\kappa=\frac{2}{3} \frac{K_{3}}{\beta_{H} K_{2}^{2}},
\end{gathered}
$$

where $\left(x, x^{\prime}\right)$ are obtained from the Courant-Snyder [6] coordinates $\left(X, X^{\prime}\right)$ by means of the nonsymplectic transformations

$$
\mathbf{x}=\frac{K_{2} \beta_{H}^{3 / 2}}{2} \mathbf{X} \quad \text { with } K_{l}=\frac{L}{B_{0} \rho} \frac{\partial^{l} B_{y}}{\partial x^{l}},
$$

$K_{2}\left(K_{3}\right)$ being the integrated sextupole (octupole) gradient, $L$ being the length of the nonlinear element, $B_{y}$ being the vertical component of the magnetic field, and $B_{0} \rho$ being the magnetic rigidity of the charged particle. Here, $\beta_{H}$ is the value of the horizontal beta-function at the sextupole location. $R\left(2 \pi \nu_{n}\right)$ is a $2 \times 2$ rotation matrix of angle $\nu_{n}$ (the fractional part of the tune).

The map (1) is actually a time-dependent system through the linear tune. The importance of introducing a time dependence is twofold. First, it allows varying the phase space topology, thus creating and moving the islands. Second, it allows trapping particles inside the islands, which is the necessary condition for the proposed scheme to work efficiently. In fact, a classical time-independent 2D system always has an invariant of motion, namely, a scalar quantity satisfying $I\left(\mathbf{x}_{n}\right)=I\left(\mathbf{x}_{0}\right)$. This is certainly the case for the linearized version of the map (1) $\left[I(\mathbf{x})=x^{2}+x^{12}\right]$, and, even in the nonlinear case, an approximate invariant can be constructed, e.g., by using the normal form approach [5]. This implies that a particle outside an island would never be able to jump across the separatrix. A slow variation of the linear tune, adiabatic with respect to the time scale introduced by the betatron oscillations, allows particles to cross the separatrix and be trapped inside the newly created islands.

In the analysis reported here, the function representing the tune variation is shown in Fig. 2. In the first part, the linear tune is decreased linearly: the capture process takes place here. Then a zero-slope part follows, used to allow the beam to filament after capture, to match better the phase space topology. Finally, a second linear decrease is

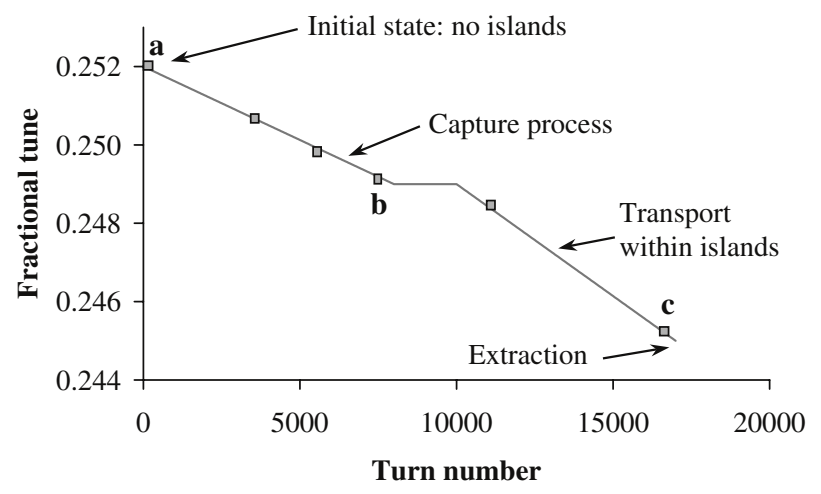

FIG. 2. Tune as a function of turn number $n$ used for the model (1). The points on the curve labeled with $\mathbf{a}, \mathbf{b}$, and $\mathbf{c}$ correspond to the values of $\nu_{n}$ used to generate the phase space portraits shown in Fig. 3.

performed which allows the islands to be moved towards higher amplitudes before extraction.

The different stages of this novel extraction are shown in Figs. 3(a)-3(c), evaluated by the computer code GIOTTO [7]. Each orbit consists of a set of points generated by the map (1) where, for this special purpose, the linear tune has been kept constant, namely, $\mathbf{x}_{j}=\mathbf{M}^{j}\left(\mathbf{x}_{0}\right), 1 \leq j \leq$ $2 \times 10^{3}$, and different orbits correspond to different initial conditions $\mathbf{x}_{0}$ with $\kappa=-2$.

In Fig. 3(a), the phase portrait for $\nu=0.252$ is shown. The phase space is foliated in closed curves and no chain of islands is visible, apart from an eleventh-order one near the dynamic aperture. This configuration corresponds to the initial state, where the beam is located around the origin and the dynamics is quasilinear. In Fig. 3(b) the topology of the phase space is shown for $\nu=0.2492$. A chain of four islands is clearly visible near the origin. An important feature is that, at higher amplitude, the phase space is again foliated into closed curves until the last regular curve that corresponds to the border of the dynamic aperture. Under these conditions, it is possible to split the beam into five slices: one around the origin and four inside the stable islands. The closed curve outside the chain acts as a barrier, preventing particles from moving towards higher amplitudes. Finally, Fig. 3(c) represents the phase space topology for $\nu=0.2453$. The four islands are still present, but their amplitude is increased. This means that particles trapped inside islands can be transported towards the outside of phase space.

The whole process has been simulated by using the model (1) with $\kappa=-2$, while the tune $\nu_{n}$ is varied according to the curve shown in Fig. 2. A set of Gaussiandistributed initial conditions has been generated, and its evolution is shown in Fig. 4. The Coulomb interaction between the charged particles is not taken into account in these simulations. The trapping process is clearly visible in the picture: it generates five beam slices, well separated at the end of the process. No particle is lost during the trapping phase, or when the islands are moved. The five slices have rather similar surfaces, but also their shape 

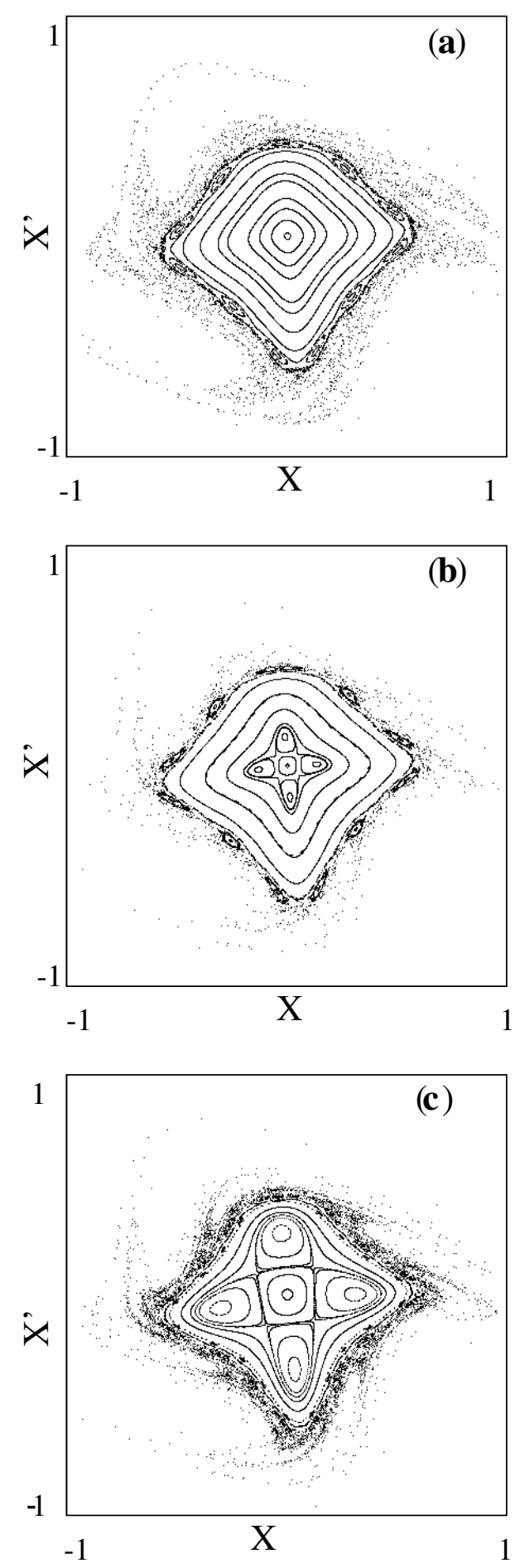

FIG. 3. Phase space portrait of the map (1) for $\nu=0.252$ (a), $\nu=0.2492(\mathrm{~b})$, and $\nu=0.2453$ (c).

matches the phase space topology very well. The first four extracted beam slices have exactly the same emittance, as their shape is dictated by the same phase space structure, i.e., the island along the positive $x$ axis. In this respect, the novel approach proves to be superior to the present CT extraction mode.

The distribution functions $\rho(x)$ for the different beam slices at the end of the trapping and transport process are
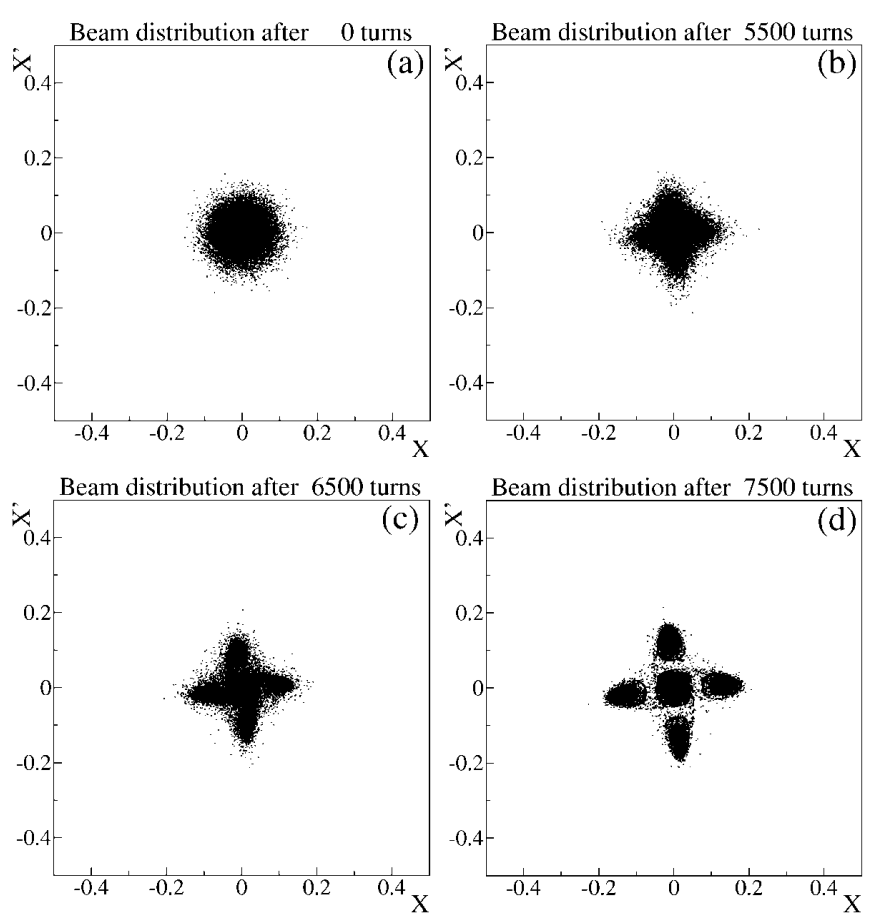

Beam distribution after 7500 turns
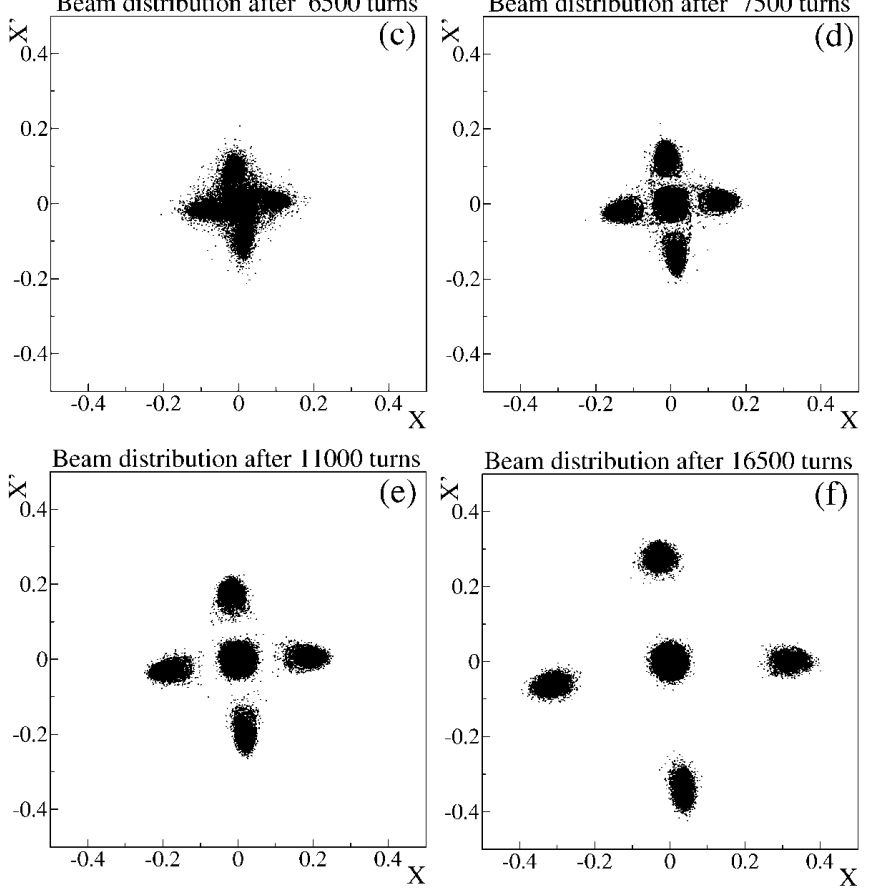

FIG. 4. Evolution of the beam distribution during the trapping process. The different plots [(a)-(f)] correspond to tune values represented by solid squares in Fig. 2. Each plot represents $2.25 \times 10^{4}$ points. The initial Gaussian distribution is centered on zero and has $\sigma=0.04$.

shown in Fig. 5. They are obtained from the results of the numerical simulations by summing all the contributions from the angular variable $x^{\prime}$. They are all Gaussian-like, thus showing that the shape is almost preserved throughout the whole process. Additional numerical simulations have been carried out to test the sensitivity of the proposed scheme to the functional dependence of the tune $\nu$ on $n$ and on the strength of the nonlinearity $\kappa$. Power laws of $\nu_{n}$, namely, $\nu_{n}=\nu_{a}+\left(\nu_{b}-\nu_{a}\right)\left(\frac{n-n_{0}}{n_{1}}\right)^{m}, n_{0} \leq n \leq$ $n_{0}+n_{1}$ have been simulated with $1 \leq m \leq 3$ : no major effects in the properties of the captured beam were observed, provided the tune variation was slow when the islands approach the center of phase space. As far as the dependence on $\kappa$ is concerned, numerical simulations confirm that the results do not change when $\kappa$ is varied by $\pm 20 \%$.

The results of the numerical simulations presented in this paper, although rather encouraging, are only the first 


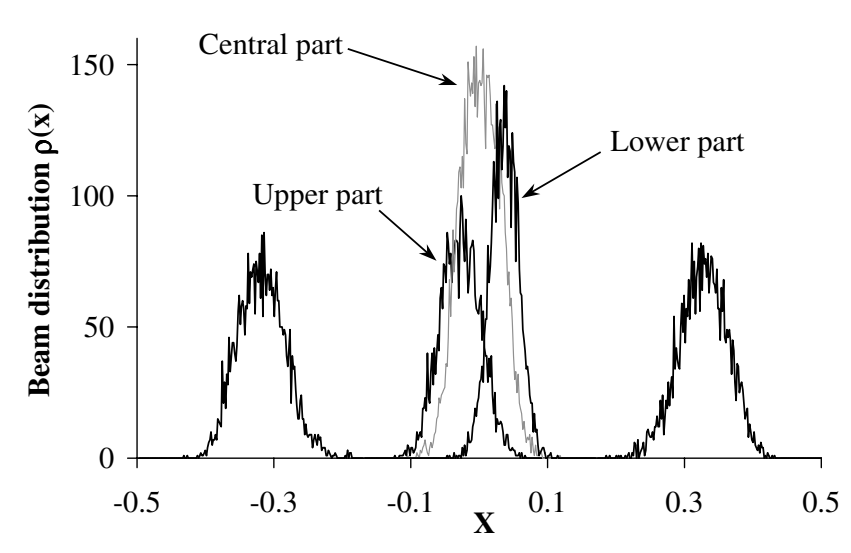

FIG. 5. Beam distribution function $\rho(x)$ at the end of the capture and transport process, for all five beam slices.

step towards a well-established technique for multiturn extraction. It has to be stressed that the method presented in this paper is completely general. However, for highintensity beams, one could question whether the proposed approach would work. The space-charge forces generate a tune shift. The coherent part can be neglected, as it represents a tune offset of the beam as a whole. The incoherent one is given by (see Refs. [8,9] for more details)

$$
\begin{aligned}
\Delta \nu_{\text {inc }}= & -\frac{2 r_{p}}{e c} \frac{R I_{0}}{\beta^{3} \gamma^{3}}\left\langle\beta_{H}\right\rangle \\
& \times\left[\frac{\epsilon_{s c}}{b^{2}} \frac{1}{B_{f}}+\frac{\epsilon_{1}}{h^{2}}\left(\beta^{2} \gamma^{2}+\frac{1}{B_{f}}\right)\right],
\end{aligned}
$$

where $r_{p}, e, c, \beta, \gamma$ are the classical proton radius, electron charge, speed of light, and relativistic factors, respectively. Here, $R,\left\langle\beta_{H}\right\rangle, I_{0}$, and $B_{f}$ represent the machine radius, the average beta-function, the average beam current, and the bunching factor (ratio of average to peak current). Finally, $\epsilon_{s c}, \epsilon_{1}$ are the Laslett coefficients: they depend on the beam size and vacuum chamber size. By assuming Gaussian beams, total intensity of $3 \times 10^{13}$, and typical PS machine parameters, $\Delta \nu_{\text {inc }}$ is either $-2.8 \times$ $10^{-3}$ or $-2.4 \times 10^{-4}$, depending on the momentum of the extracted beam, i.e., 14 or $26 \mathrm{GeV} / c$, respectively. For
$26 \mathrm{GeV} / c$, the space-charge tune shift is negligible. For $14 \mathrm{GeV} / c$, the space-charge tune shift, which is about onethird of the total tune variation needed to capture the beam, can no longer be neglected. However, it has to be stressed that $\Delta \nu_{\text {inc }}$ represents the maximum tune shift, achieved near the beam center. Particles at higher amplitude experience a smaller tune shift. The adiabatic trapping is actually performed by capturing particles at higher amplitudes: those near the beam center are left unperturbed. Hence, it seems reasonable to assume that the process should not be very much affected, even in this case.

Other points also need clarifying. The quantitative relationship between the transverse emittance of the different slices and the island parameters should be found. Also, the link between the way $\nu_{n}$ is varied and the trapping efficiency should be quantified to optimize the adiabatic capture. The analysis of these two points would allow good control of the transverse properties of the extracted beam to be achieved. Finally, the stability of this method by introducing full $4 \mathrm{D}$ betatron motion as well as chromaticity effects should be checked in detail.

Experiments using the PS proton beam are already planned to verify the practical feasibility of this novel extraction method.

[1] M. M. Gordon and T. A. Welton, Bull. Am. Phys. Soc. 3, 57 (1958).

[2] K. Hiramoto and M. Nishi, Nucl. Instrum. Methods Phys. Res., Sect. A 322, 154 (1992).

[3] C. Bovet et al., in Proceedings of the 1973 Particle Accelerator Conference, edited by D. W. Dupen (IEEE, New York, 1973), p. 438.

[4] CERN Report No. 98-02, edited by K. Elsener et al., 1998 (unpublished).

[5] A. Bazzani et al., CERN Yellow Report No. 94-02, 1994 (unpublished).

[6] E. Courant and H. Snyder, Ann. Phys. (Paris) 3, 1 (1958).

[7] D. Bortolotti et al., Int. J. Mod. Phys. C 6, 651 (1995).

[8] L. J. Laslett, BNL Report No. 7534, 1963 (unpublished), p. 324-367.

[9] B. Zotter, CERN ISR-TH Report No. 72-8, 1972 (unpublished). 